Article

\title{
Channel Characteristics of InAs/AlSb Heterojunction Epitaxy: Comparative Study on Epitaxies with Different Thickness of InAs Channel and AlSb Upper Barrier
}

\author{
He Guan ${ }^{1}$, Shaoxi Wang ${ }^{1} * \mathbb{D}$, Lingli Chen ${ }^{1}$, Bo Gao ${ }^{2}$, Ying Wang ${ }^{1} \mathbb{D}$ and Chengyu Jiang ${ }^{1}$ \\ 1 School of Microelectronics, Northwestern Polytechnical University (NPU), Xi'an 710072, China; \\ he.guan@nwpu.edu.cn (H.G.); llchen27@126.com (L.C.); yingwang@nwpu.edu.cn (Y.W.); \\ 2013301856@mail.nwpu.edu.cn (C.J.) \\ 2 Xi'an Microelectronics Technology Institute, Xi'an 710000, China; bobbygoff@foxmail.com \\ * Correspondence: shxwang@nwpu.edu.cn
}

Received: 11 April 2019; Accepted: 9 May 2019; Published: 13 May 2019

\begin{abstract}
Because of the high electron mobility and electron velocity in the channel, InAs/AlSb high electron mobility transistors (HEMTs) have excellent physical properties, compared with the other traditional III-V semiconductor components, such as ultra-high cut-off frequency, very low power consumption and good noise performance. In this paper, both the structure and working principle of InAs/AlSb HEMTs were studied, the energy band distribution of the InAs/AlSb heterojunction epitaxy was analyzed, and the generation mechanism and scattering mechanism of two-dimensional electron gas (2DEG) in InAs channel were demonstrated, based on the software simulation in detail. In order to discuss the impact of different epitaxial structures on the 2DEG and electron mobility in channel, four kinds of epitaxies with different thickness of InAs channel and AlSb upper-barrier were manufactured. The samples were evaluated with the contact Hall test. It is found the sample with a channel thickness of $15 \mathrm{~nm}$ and upper-barrier layer of $17 \mathrm{~nm}$ shows a best compromised sheet carrier concentration of $2.56 \times 10^{12} \mathrm{~cm}^{-2}$ and electron mobility of $1.81 \times 10^{4} \mathrm{~cm}^{2} / \mathrm{V} \cdot \mathrm{s}$, and a low sheet resistivity of $135 \Omega / \square$, which we considered to be the optimized thickness of channel layer and upper-barrier layer. This study is a reference to further design InAs/AlSb HEMT, by ensuring a good device performance.
\end{abstract}

Keywords: InAs/AlSb heterojunction; epitaxy; 2DEG; electron mobility

\section{Introduction}

Compared with traditional III-V semiconductor materials such as GaAs, InP and $\mathrm{GaN}$, antimonide-based compound semiconductors (ABCS) have higher electron mobility and electron velocity, which present them with broad prospects in ultra-high speed, low power consumption and low noise applications. InAs with a lattice constant of about $6.05 \mathrm{eV}$ is a typical ABCS material. Its electron mobility is 3 and 5 times of that of GaAs and InP materials, respectively; its electron saturation drift velocity is about 5 times of that of GaAs and InP materials; its effective mass of electrons is $1 / 3$ of that of GaAs and InP materials; especially, its bandgap is only $0.35 \mathrm{eV}$, and is much more narrow than GaN, which makes InAs have excellent electrical properties at very low bias voltage. So InAs is often used as channel material for high-speed and low-power-consumption HEMT.

As another typical antimony material, AlSb has little lattice mismatch with InAs, but its band gap of $1.27 \mathrm{eV}$ with InAs can form very deep electron potential wells, and makes the InAs/AlSb heterogeneous structures possess a high concentration of two-dimensional electron gas density (2DEG). 
Therefore, InAs/AlSb high electron mobility transistors (HEMTs) devices, with AlSb as a barrier layer and InAs as the channel layer, have excellent physical properties, such as ultra-high cut-off frequency, very low power consumption and good noise performance, and present a very good application prospect in analog/digital circuit, microwave field and space communication [1-5]. As we know, the properties of HEMTs are fundamentally determined by the performance of epitaxial materials. So far, lots of research on III-V epitaxial materials focus on GaN/AlGaN, InAlAs/InGaAs, etc. [6-8]. However, studies on InAs/AlSb epitaxial materials are not broad, with few results [9-12]. Therefore, a further research on the structures of epitaxial materials is significant to deeply understand the performance of devices. In this paper, the structure and working principle of InAs/AlSb HEMTs were studied, the energy band distribution of the InAs/AlSb heterojunction epitaxy was analyzed, and the generation mechanism and scattering mechanism of two-dimensional electron gas (2DEG) in the InAs channel were demonstrated by simulation in detail; additionally, four kinds of epitaxy samples with different thicknesses of InAs channel and AlSb upper barrier were fabricated and tested, in order to discuss their channel characteristics.

\section{Simulation and Principle Analysis}

In order to study the working principle and performance of the InAs/AlSb heterojunction in detail, a simulation is applied based on the typical device structure as shown in Figure 1 [4,13,14]. A $200 \mathrm{~nm}$ GaAs material is settled as substrate. The lattice constant of GaAs as $5.653 \AA$ presents a significant deviation from the value of InAs as $6.058 \AA$, leading to a lattice mismatch degree of 7.1\%. This mismatch makes it easy to generate defects. Therefore, a $700 \mathrm{~nm}$ AlGaSb layer with a lattice constant of $6.1 \AA$, chosen as a buffer to release the mismatch, is settled on GaAs substrate. Then an AlSb/InAs/AlSb laminated layer as a quantum well structure is settled to form the down-barriers/channel/upper-barriers. InAs forms the channel and the 2DEG is located on the side of the InAs channel near the InAs/AlSb (upper-barrier) heterojunction. An Si-doped InAs layer with a $10^{19} \mathrm{~cm}^{-3}$ doping density is inserted into the AlSb upper-barrier layer in order to make the semiconductor exhibit as an n-type semiconductor to completely ionize, in order to form positive ionized donors and free electrons. Because the band-gap width of InAs is much smaller than AlSb, leading electrons in the heavy doped n-type AlSb to shift from the AlSb region, where it is with higher energy, to the non-doped InAs channel, where it is with lower electron energy. This effect makes the electrons accumulating on the opposite side of the InAs channel to form 2DEG. Meanwhile, these positive ionized donors are left on the AlSb side, making a space charge zone formed at the junction to induce an electric field. This electric field causes the band to bend.

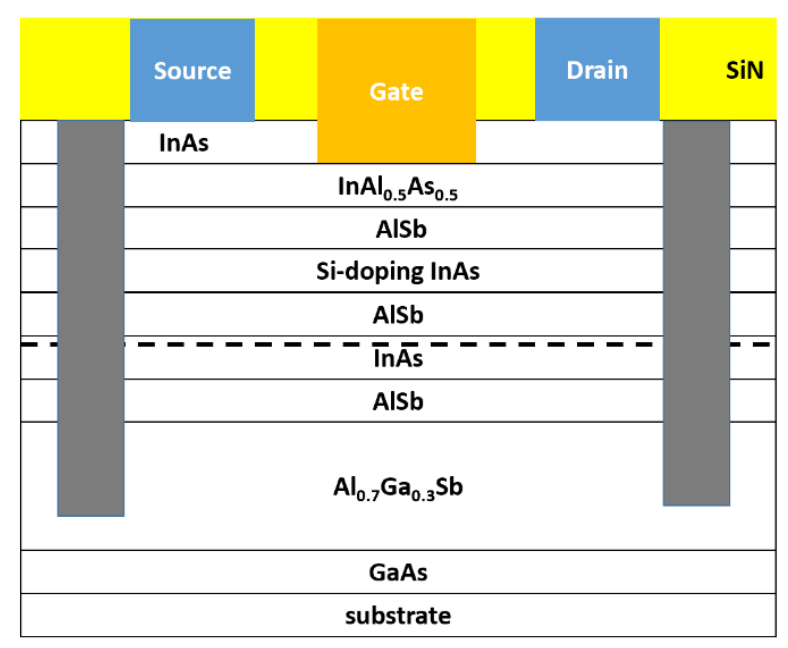

Figure 1. Structure of InAs/AlSb high electron mobility transistor (HEMT). 
Therefore, the modulation-doped structure formed by heavily doped n-type wide-band-gap semiconductor (AlSb region), and no-doping narrow-band-gap semiconductor (InAs region), can ensure the electron supply process happening in AlSb area, and the electron transport process happening in the InAs channel. These two processes are separated in space, which can effectively eliminate the impact of ionized impurity scattering during electrons transportation, and greatly improve the electron mobility and 2DEG in the channel [15-17]. Finally, an InAlAs protection layer is settled to reduce hole gate leakage current, and an InAs layer is settled as the cap layer. A heavy doping was applied on the ohmic contact region of InAs cap layer in order to reduce the contact resistant [18]. A Schottky contact forms between the gate metal electrode and the InAlAs protection layer [19], to prevent the electrons continually spreading to the metal. Controlling the gate bias voltage can change the Schottky barrier, thus controlling the 2DEG density in channel.

In this paper, we used Sentaurus Technology Computer Aided Design (TCAD) (2010 version, Synopsys Inc., Mountain View, CA, USA) to study the generation mechanism and scattering mechanism of 2DEG in the InAs channel [20]. In the simulation, the hydrodynamic model was chosen for electron transport, the drift-diffusion model was chosen for hole transport, the high-field-saturation mobility was chosen to be the mobility model, and the SRH recombination module was chosen to be the generation-recombination module. The simulated result of the conduction band of InAs/AlSb (upper-barrier) heterojunction is shown in Figure 2. It is found that a triangle electronic potential well is indeed formed near the heterojunction. AlSb with higher barrier height works well to limit the electrons in the InAs channel in order to form a high 2DEG.

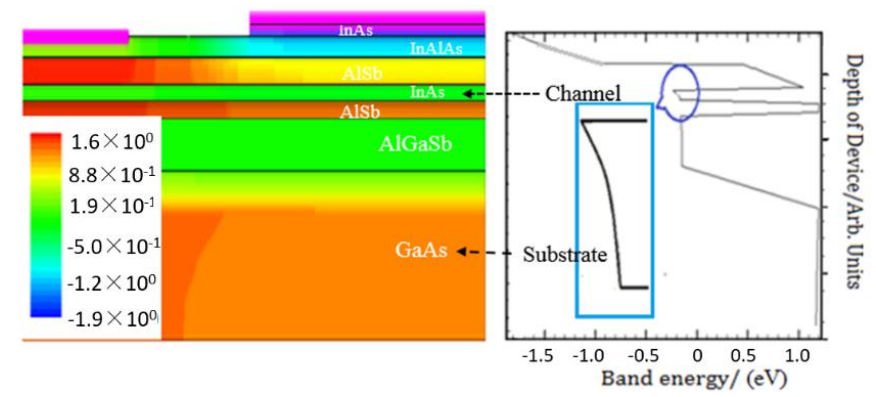

Figure 2. Longitudinal distribution of conductive band in heterojunction zone from simulation.

The key parameters of epitaxy such as 2DEG density, electron mobility and electron velocity were simulated as well. As shown in Figure 3, the maximum electron concentration in the channel reaches to the order of $10^{19} \mathrm{~cm}^{-3}$. The sheet carrier concentration which can be obtained by integrating the curve of electron concentration is generally at the order of $10^{12} \mathrm{~cm}^{-2}$. The electron mobility at the channel is simulated as shown in Figure 4. It is found the electron mobility on the side of the InAs channel near the AlSb upper-barrier presents a relatively high value with the order of $10^{4} \mathrm{~cm}^{2} / \mathrm{V} \cdot \mathrm{s}$, which is obviously higher than the other side of the InAs channel. This is consistent with the region of 2DEG location that we analyzed above.

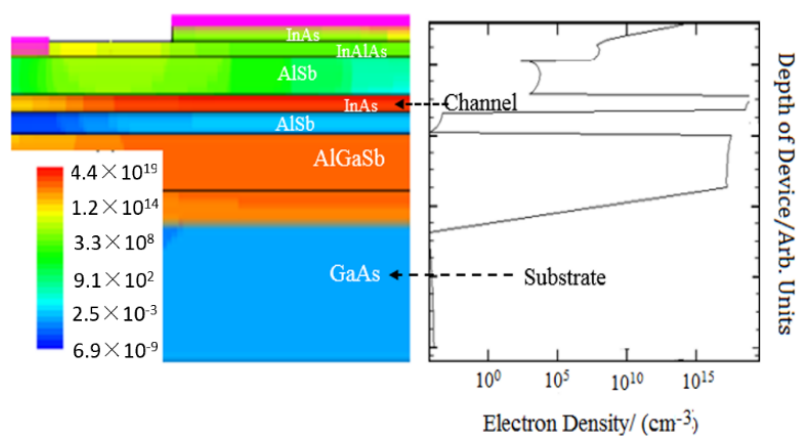

Figure 3. Distribution of electron concentration from simulation. 


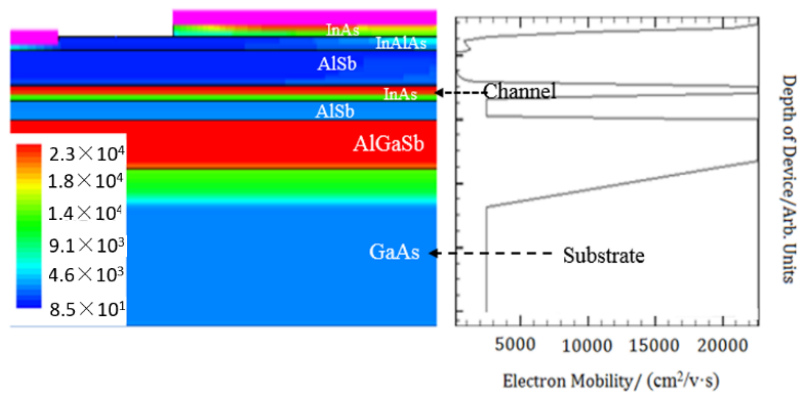

Figure 4. Distribution of electron mobility from simulation.

As can be seen in Figure 5, the electronic velocity is stable in the channel, but appears an increase in the area of the AlSb upper barrier near the source and drain. This is due to the high field strength formation between the ionized donors in the upper barrier and the electrode. Electron concentration in this part is not large, but electrons would be instantly accelerated to higher speed under the high field strength.

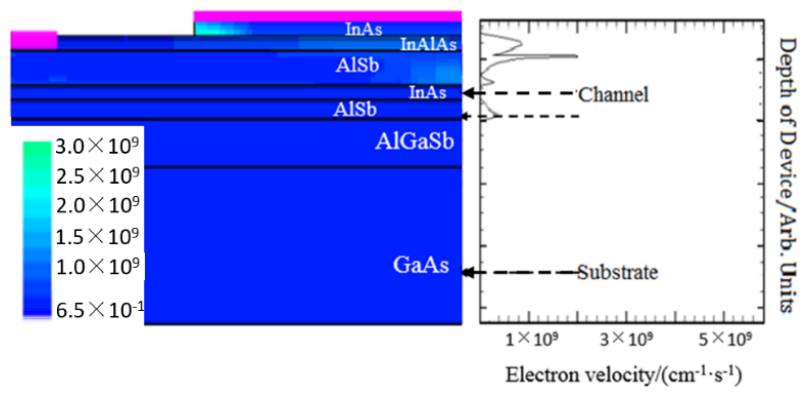

Figure 5. Distribution of electron velocity from simulation.

\section{Experiments and Analysis}

Four kinds of InAs/AlSb epitaxies with different thicknesses of InAs channel layer and AlSb upper-barrier layer were manufactured in this paper. Sample \#1, with the structure in Figure 6a as the same as the epitaxy module in above simulation, is to be the reference structure: The InAs channel is set with $15 \mathrm{~nm}$, and the AlSb upper barrier is set with $15 \mathrm{~nm}$ (the part below Si-doping InAs thin film is $5 \mathrm{~nm}$ ). In order to verify the impact of channel thickness, the sample \#2, with a reduced channel thickness of $12 \mathrm{~nm}$, is designed as in Figure 6b; in order to explore the impact of the thickness of the AlSb upper barrier layer, 17 and $13 \mathrm{~nm}$ upper barriers are applied in sample \#3 and sample \#4, respectively, and their structures are as shown in Figure $6 c, d$.

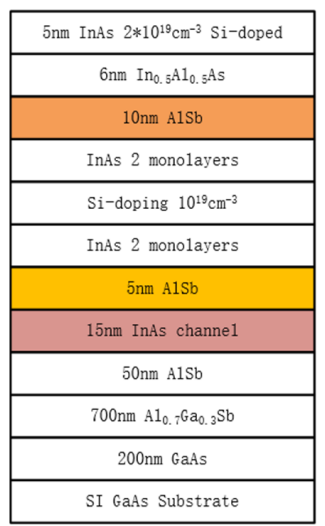

(a)

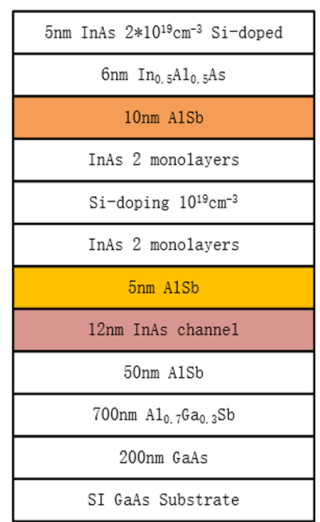

(b)

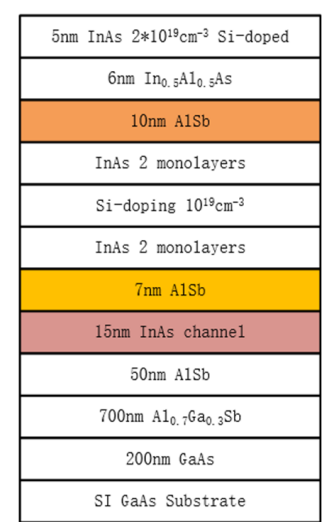

(c)

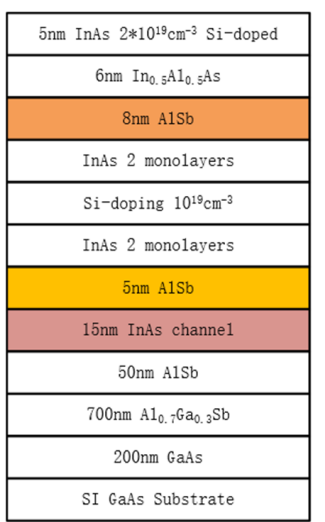

(d)

Figure 6. Epitaxy structure diagram of 4 different samples: (a) is for Sample \#1, (b) is for Sample \#2, (c) is for Sample \#3, and (d) is for Sample \#4. 
Molecular beam epitaxy (MBE) was chosen to grow the epitaxy in this study [21]. First, a semi-insulated GaAs substrate was treated by de-oxygenation, then a GaAs buffer layer with a thickness of $200 \mathrm{~nm}$ was grown at 610 degree centigrade. A $700 \mathrm{~nm}$ AlGaSb layer was grown on the GaAs epitaxial layer to be the buffer under 580 degrees centigrade. Subsequently, we cooled the temperature to 540 degrees centigrade to grow the AlSb/InAs/AlSb layer, including inserting a thin Si-doping InAs film with four atomic layer thickness in the AlSb upper barrier layer. Finally, the InAlAs protection layer and InAs cap layer was grown at 540 degree centigrade, as well. The surface is observed by atomic force microscope (AFM) with the test area of $10 \mu \mathrm{m} \times 10 \mu \mathrm{m}$. The AFM graph is shown in Figure 7. It is found that the surface roughness is good, and the Root-mean-square value of surface roughness (RMS) is tested as around $1.4 \mathrm{~nm}$, as shown in Table 1. It indicates that the epitaxy is grown with a good compactness and uniformity.

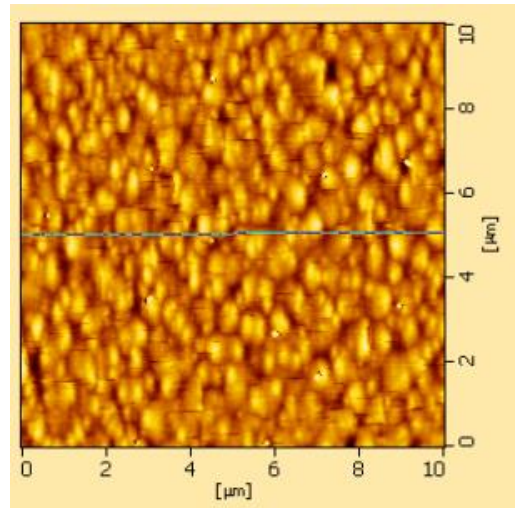

(a)

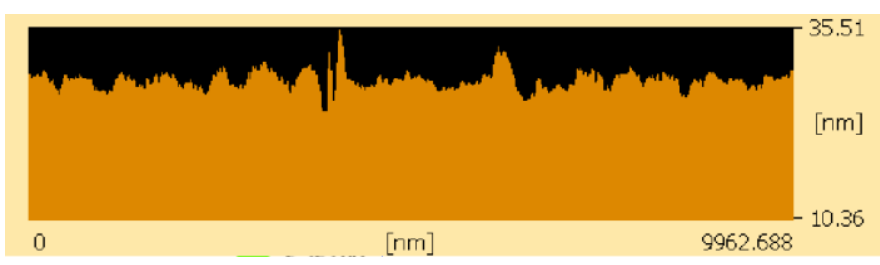

(b)

Figure 7. Atomic force microscope (AFM) test result: (a) Photograph of the surface; (b) Surface longitudinal photograph.

Table 1. RMA value of the four samples.

\begin{tabular}{cc}
\hline Sample Number & RMS (nm) \\
\hline$\# 1$ & 1.432 \\
$\# 2$ & 1.328 \\
$\# 3$ & 1.435 \\
$\# 4$ & 1.394 \\
\hline
\end{tabular}

The samples were evaluated with the contact Hall test. Four indium points were added on the surface of the epitaxy to be the external electrodes, and the performance parameters were tested. The test data of the four samples are summarized in Table 2. It is found that the mobility and sheet carrier concentration vary obviously. The sample \#1 presents a sheet carrier concentration of $2.57 \times 10^{12} \mathrm{~cm}^{-2}$ and an electron mobility of $1.58 \times 10^{4} \mathrm{~cm}^{2} / \mathrm{V} \cdot \mathrm{s}$. The sample \#2 shows an obviously increased mobility of $1.71 \times 10^{4} \mathrm{~cm}^{2} / \mathrm{V} \cdot \mathrm{s}$. This is because the reduced thickness of the channel would suppress the lattice mismatch dislocation, leading to a reduced interfacial scattering, thus the mobility of channel carriers is enhanced. However, it shows the lowest sheet carrier concentration of $2.29 \times 10^{12} \mathrm{~cm}^{-2}$, because the reduced thickness of channel layer causes the quantum well energy level to increase, and quantum well depth to decrease, thus the 2DEG density is reduced. The electron mobility of sample \#4 is relatively lowest, the reason is that the upper barrier layer is much thinner, and the scattering of impurities on the interface is enhanced, so the electron velocity is degraded. Sample \#3 shows a compromised sheet carrier concentration of $2.56 \times 10^{12} \mathrm{~cm}^{-2}$ and electron mobility of $1.81 \times 10^{4} \mathrm{~cm} / \mathrm{V} \cdot \mathrm{s}$, and the lowest sheet resistivity of $135 \Omega / \square$. It is found that the 2 DEG density and electron mobility are conflict parameters in general, i.e., an increased 2DEG density always accompanies a decreased electron mobility. This can be explained as following: The volume of the electrons penetrating to the AlSb/InAs 
interface would increase if the 2DEG density increases, which would induce the chaos and confusion of the lattice arrangement in the interface, thus making the interface roughness scattering effect more obvious. In this condition, electron momentum relaxation would occur at the interface, and the direction and speed of the electron motion would change. Therefore, the electron mobility decreases significantly. This indicates that it is difficult to obtain the most optimal value of 2DEG and electron mobility at the same time, and it is necessary to compromise the two parameters in real applications.

Table 2. Hall test result of the four kinds of samples.

\begin{tabular}{cccc}
\hline $\begin{array}{c}\text { Sample } \\
\text { Number }\end{array}$ & $\begin{array}{c}\text { Sheet Carrier Concentration } \\
\left(\mathbf{c m}^{-\mathbf{2}} \mathbf{)}\right.\end{array}$ & $\begin{array}{c}\text { Electron Mobility } \\
\left(\mathbf{c m}^{\mathbf{2}} \mathbf{/ V} \cdot \mathbf{s}\right)\end{array}$ & $\begin{array}{c}\text { Sheet Resistivity } \\
{[\Omega / \square]}\end{array}$ \\
\hline$\# 1$ & $2.57 \times 10^{12}$ & 15776.53 & 154 \\
$\# 2$ & $2.29 \times 10^{12}$ & 17101.36 & 159 \\
$\# 3$ & $2.56 \times 10^{12}$ & 18088.44 & 135 \\
$\# 4$ & $2.81 \times 10^{12}$ & 14312.70 & 155 \\
\hline
\end{tabular}

\section{Conclusion}

The thickness of InAs channel and AlSb barrier layer obviously impact the performance of the InAs/AlSb heterojunction epitaxy. In general, the 2DEG density and electron mobility are conflict parameters. When the channel thickness is decreased from 15 to $12 \mathrm{~nm}$, electron mobility is reduced from $1.77 \times 10^{4}$ to $1.51 \times 10^{4} \mathrm{~cm}^{2} / \mathrm{V} \cdot \mathrm{s}$. When the thickness of upper barrier is reduced from 15 to $13 \mathrm{~nm}$, the sheet carrier concentration increases from $2.57 \times 10^{12}$ to $2.91 \times 10^{12} \mathrm{~cm}^{-2}$; however, electron mobility decreased obviously to $1.43 \times 10^{4} \mathrm{~cm}^{2} / \mathrm{V} \cdot \mathrm{s}$; conversely, when the thickness of the AlSb upper barrier layer is increased from 15 to $17 \mathrm{~nm}$, the device shows a compromised sheet carrier concentration of $2.56 \times 10^{12} \mathrm{~cm}^{-2}$ and an electron mobility of $1.81 \times 10^{4} \mathrm{~cm}^{2} / \mathrm{V} \cdot \mathrm{s}$, and a low sheet resistivity of $135 \Omega / \square$. Therefore, we choose the channel thickness of $15 \mathrm{~nm}$, and the upper-barrier layer of $17 \mathrm{~nm}$, to be the optimized thickness, which is a reference to further design InAs/AlSb HEMT, by ensuring a good device performance.

Author Contributions: Formal analysis, investigation, and writing-original draft preparation, H.G.; software, L.C.; writing-review and editing, S.W., B.G., and Y.W.; data curation, C.J.

Funding: This research was funded by "National science foundation of Shaanxi province, China, grant number 2018JQ6069" and "China Postdoctoral Science Foundation, grant number 2018M643733".

Acknowledgments: We are appreciated the support of Lv Hongliang from Xi Dian University of China on the experiment data.

Conflicts of Interest: The authors declare no conflict of interest.

\section{References}

1. Moschetti, G.; Wadefalk, N.; Desplanque, L.; Wallart, X.; Grahn, J.; Nilsson, P.-Å.; Abbasi, M. Cryogenic InAs/AlSb HEMT wideband low-noise IF amplifier for ultra-low-power applications. IEEE Microw. Wirel. Compon. Lett. 2012, 22, 144-146. [CrossRef]

2. Guan, H.; Lv, H.; Guo, H.; Zhang, Y. Small-signal modeling with direct parameter extraction for impact ionization effect in high-electron-mobility transistors. J. Appl. Phys. 2015, 118, 195702. [CrossRef]

3. Guan, H.; Jiang, C. Study on the physical and leakage current characteristics of an optimized high-k/InAlAs MOS capacitor with a $\mathrm{HfO}_{2}-\mathrm{Al}_{2} \mathrm{O}_{3}$ laminated dielectric. Coatings 2018, 8, 417. [CrossRef]

4. Guan, H.; Lv, H.; Zhang, Y.; Zhang, Y. Improved modeling on the RF behavior of InAs/AlSb HEMTs. Solid State Electron. 2015, 114, 155-162. [CrossRef]

5. Bennett, B.R.; Magno, R.; Boos, J.B.; Kruppa, W.; Ancona, M.G. Antimonide-based compound semiconductors for electronic devices: A review. Solid State Electron. 2005, 49, 1875-1895. [CrossRef]

6. Chvála, A.; Nagy, L.; Marek, J.; Priesol, J.; Donoval, D.; Blaho, M.; Gregušová, D.; Kuzmík, J.; Šatka, A. Characterization of monolithic InAIN/GaN NAND logic cell supported by circuit and device simulations. IEEE Trans. Electron Device. 2018, 65, 2666-2669. [CrossRef] 
7. Begum, M.S.; Vijayashree, J.; Mohanbabu, A.; Mohankumar, N. Investigation of performance of InAsSb based high electron mobility transistors (HEMTs). In Proceedings of the 2017 Devices for Integrated Circuit (DevIC), Kalyani, India, 23-24 March 2017; IEEE: Piscataway, NJ, USA, 2017. [CrossRef]

8. Shrestha, N.M.; Li, Y.; Suemitsu, T.; Samukawa, S. Electrical characteristic of AlGaN/GaN high-electronmobility transistors with recess gate structure. IEEE Trans. Electron Device. 2019, 66, 1694-1698. [CrossRef]

9. Maleev, N.A.; Bobrov, M.A.; Kuzmenkov, A.G.; Vasil'ev, A.P.; Kulagina, M.M.; Maleev, S.N.; Blokhin, S.A.; Nevedomsky, V.N.; Ustinov, V.M. Epitaxial InGaAs/InAlAs/AlAs structures for heterobarrier varactors with low leakage current. Tech. Phys. Lett. 2018, 44, 862-864. [CrossRef]

10. Hopkins, P.F.; Rimberg, A.J.; Westervelt, R.M.; Tuttle, G.; Kroemer, H. Quantum Hall effect in InAs/AlSb quantum wells. Appl. Phys. Lett. 1991, 58, 1428-1430. [CrossRef]

11. Zhang, J.; Lv, H.; Ni, H.; Niu, Z.; Zhang, Y. Temperature dependence on the electrical and physical performance of InAs/AlSb heterojunction and high electron mobility transistors. Chin. Phys. B 2018, 27, 097201. [CrossRef]

12. Bolognesi, C.R.; Kroemer, H.; English, J.H. Well width dependence of electron transport in molecular-beam epitaxially grown InAs/AlSb quantum wells. J. Vac. Sci. Technol. B 1992, 10, 877-879. [CrossRef]

13. Malmkvist, M.; Lefebvre, E.; Borg, M.; Desplanque, L.; Wallart, X.; Dambrine, G.; Bollaert, S.; Grahn, J. Electrical characterization and small-signal modeling of InAs/AlSb HEMTs for low-noise and high-frequency applications. IEEE Tran. Microw. Theory Tech. 2009, 56, 2685-2691. [CrossRef]

14. Werking, J.D.; Bolognesi, C.R.; Chang, L.-D.; Nguyen, C.; Hu, E.L.; Kroemer, H. High-transconductance InAs/AlSb heterojunction field-effect transistors with delta-doped AlSb upper barriers. IEEE Electron Device Lett. 1992, 13, 164-166. [CrossRef]

15. Baraskar, A.; Jain, V.; Wistey, M.A.; Singisetti, U.; Lee, Y.J.; Thibeault, B.; Gossard, A.; Rodwell, M.J. High doping effects on in-situ Ohmic contacts to n-InAs. In Proceedings of the International Conference on Indium Phosphide and Related Materials (IPRM), Kagawa, Japan, 31 May-4 June 2010; IEEE: Piscataway, NJ, USA, 2010. [CrossRef]

16. Lin, H.K.; Kadow, C.; Dahlström, M.; Bae, J.U.; Rodwell, M.J.W.; Gossard, A.C.; Brar, B.; Sullivan, G.; Nagy, G.; Bergman, J. InAs/InAsP composite channels for antimonide-based field-effect transistors. Appl. Phys. Lett. 2004, 84, 437-439. [CrossRef]

17. Lin, H.K.; Kadow, C.; Bae, J.U.; Rodwell, M.J.W.; Gossard, A.C.; Brar, B.; Sullivan, G.; Nagy, G.; Bergman, J. Design and characteristics of strained InAs/InAlAs composite channel heterostructure field-effect transistors. J. Appl. Phys. 2005, 97, 024505. [CrossRef]

18. Dormaier, R.; Zhang, Q.; Liu, B.; Chou, Y.C.; Lange, M.D.; Yang, J.M.; Oki, A.K.; Mohney, S.E. Thermal stability of $\mathrm{Pd} / \mathrm{Pt} / \mathrm{Au}$ Ohmic contacts to InAlSb/InAs heterostructures for high electron mobility transistors. J. Appl. Phys. 2009, 105, 044505. [CrossRef]

19. Yang, M.J.; Bennett, B.R.; Fatemi, M.; Lin-Chung, P.J.; Moore, W.J.; Yang, C.H. Photoluminescence of single quantum wells: Transition from type-II to type-I band alignment. J. Appl. Phys. 2000, 87, 8192-8194. [CrossRef]

20. Ilatikhameneh, H.; Ashrafi, R.; Khorasani, S. Simulation and optimization of HEMTs. In Proceedings of the International Conference on Advances in Computational Tools for Engineering Applications (ACTEA), Beirut, Lebanon, 13-15 July 2016; IEEE: Piscataway, NJ, USA, 2016. [CrossRef]

21. Bennett, B.R.; Shanabrook, B.V. Molecular beam epitaxy of Sb-based semiconductors. In Thin Films: Heteroepitaxial Systems; World Scientific: Singapore, 1999.

(C) 2019 by the authors. Licensee MDPI, Basel, Switzerland. This article is an open access article distributed under the terms and conditions of the Creative Commons Attribution (CC BY) license (http://creativecommons.org/licenses/by/4.0/). 